\title{
Frequency of perimesencephalic sub arachnoid haemorrhage in our hospital
}

\author{
Geover Joslen Lobo ${ }^{1, *}$, Madhukar Nayak ${ }^{2}$ \\ ${ }^{1}$ Assistant Professor, ${ }^{2}$ Professor, Dept. of Neurosurgery, FR Muller Medical College, Mangalore, Karnataka, India
}

*Corresponding Author:

Email: geover80@gmail.com

\begin{abstract}
Aim: Spontaneous sub arachnoid haemorrhage (SAH) is diagnosed by imaging studies which mainly is by a computerised tomography (CT) of the brain. The cause of spontaneous SAH is most commonly due to a rupture in the vascular structures supplying blood to the brain which is detected by a CT angio of the brain. This study is hence aimed at analysing the incidence and frequency of angio negative SAH in patients admitted in our hospital.

Materials and Methods: This is a retrospective and a prospective study where 32 patients were detected either with CTA or DSA or both to be having spontaneous angio negative SAH. They were clinically graded based on the WFNS scale (6) and radiologically by the Fischer score of their plain CT brain. Patients diagnosed with classical SAH and had a structural vascular abnormality were managed as per protocol of either endovascular coiling or surgical clipping. Patients who were diagnosed to have a negative angio imaging studies were given supportive treatment.

Results: During the said period 124 patients were diagnosed to have SAH at our centre. 3 of them died before any evaluation. 33 out of 121 were diagnosed to be angio negative. The male to female ratio was nearly equal with 18 female and 15 males. The ages ranged from $40-80$ years. 1 of them was subsequently diagnosed to have a p.comm a aneurysm on a repeat DSA 4 weeks later to the ictus and underwent successful endovascular coiling. Of the 32 who were angio negative on the repeat imaging done 4 weeks later, 2 developed hydrocephalus. 1 underwent right V-P Shunt but the other was put on an EVD and succumbed to sepsis. Conclusion: The entity of PMSAH had a good outcome in our study the incidence being $26.4 \%$ and the mortality was 1 (3\%) this being not directly due to the SAH. Based on the CT images and the criteria stated the patients can't be labelled PMSAH. A repeat vascular imaging at least 4 weeks after the primary ictus is necessary.
\end{abstract}

Keywords: Spontaneous sub arachnoid hemorrhage, CT angio negative brain hemorrhage.

\section{Introduction}

Spontaneous sub arachnoid haemorrhage (SAH) is a condition diagnosed by imaging studies which mainly is by a computerised tomography (CT) of the brain. The cause of spontaneous SAH is most commonly due to a rupture in the vascular structures supplying blood to the brain. It is a known fact that spontaneous SAH is a neurosurgical emergency and needs further advanced imaging studies like CT Angiogram (CTA) or a Digital Substraction Angiogram (DSA) of the brain to detect the site of rupture. ${ }^{1}$ On angiographic studies grossly $75 \%$ are due to aneurysmal rupture, $5 \%$ due to AVM rupture and in the rest no abnormality is detected. ${ }^{2}$ These patients in whom no abnormality is detected pose a challenge on the management of $\mathrm{SAH}$, as if a finding is missed the grave sequelae which are re-bleed, hydrocephalus, vasospasm and seizures can occur to which the patient may succumb or may leave the patient with a permanent neuro deficit. Literature has evidence that a repeat neuro vascular imaging after a certain time gap has detected abnormality and hence is ideal. Incidence of these angio negative SAH in our Indian population is being worked on. We encounter such presentations in patients, as our centre is a medical college and a tertiary referral centre. This study is hence aimed at analysing the incidence and frequency of angio negative SAH in patients admitted in our hospital.

\section{Materials and Methods}

Our study was a retrospective and a prospective study observed in the patients who presented to the department of neurosurgery, Fr Muller medical college hospital, Mangaluru from January 2012 to December 2017. 32 patients were detected either with CTA or DSA or both to be having spontaneous angio negative SAH. They were clinically graded based on the WFNS scale $^{6}$ and radiologically by the Fischer score of their plain CT brain. ${ }^{7}$ All patients were given intensive care in the Neuro ICU.

Patients with symptoms suggestive of SAH and supported with an immediate plain CT brain were included into the study. Children, and adults with other causes for SAH like trauma, bleeding disorders, antiplatelet therapy, brain tumours, hypertensive cortical bleeds were excluded from our study. Their pre-existing medical conditions were not considered. The investigations done were a $\mathrm{CT}$ angiogram of the brain and a Digital subtraction angiogram of the brain.

All patients presenting to us were given a neuro ICU admission. Triple ' $\mathrm{H}$ ' therapy was started. ${ }^{8}$ Patients diagnosed with classical SAH and had a structural vascular abnormality were managed as per protocol of either endoscopic coiling or surgical clipping. The decision of treatment was decided by the team in the department and the choice offered to the patients kin to decide on the modality of definitive treatment. Patients who were diagnosed to have a negative angio imaging studies were given supportive 
treatment which included anticonvulsants preferably levetiracetam, anti-edema measures, steroids, calcium channel blocker Nimodipine to relieve vasospasm, analgesics, antacids and laxatives. They were given an appointment 3-4 weeks ${ }^{9}$ later for a re-imaging. This time gap was thought appropriate as the vasospasm would be relieved, the blood products in the subarachnoid space washed away in the CSF and any clot inside the aneurysm would be cleared thereby reducing the risks and complications due to $\mathrm{SAH}$.

\section{Results}

During the said period 124 patients were diagnosed to have SAH at our centre. 3 of them died before any evaluation. 33 out of 121 were diagnosed to be angio negative. All presented with headache, 3 patients had loss of conciousness, seizures were present in 8 and 1 presented with left side hemiparesis. The male to female ratio was nearly equal with 18 female and 15 males. The ages ranged from $40-80$ years. 1 of them was subsequently diagnosed to have a p.comm a aneurysm on a repeat DSA 4 weeks later to the ictus and underwent successful endovascular coiling. Of the 32 who were angio negative on the repeat imaging done 4 weeks later, 2 developed hydrocephalus. 1 underwent right V-P Shunt but the other was put on an EVD and succumbed to sepsis. 1 patient presented to the casuality with headache was diagnosed to have a rebleed on a plain CT brain and re-evaluated but found to be angio negative, patient was managed conservatively based on the WFNS scale, 20 were grade I, 11 were grade II, and 1 was grade III. (Table 1)

\section{Table 1:}

\begin{tabular}{|l|c|c|}
\hline & Results & \\
\hline Total no. & 32 & $26.4 \%$ \\
\hline Male & 14 & \\
\hline Female & 18 & \\
\hline WFNS scale & & \\
\hline Grade I & $\mathrm{n}=20$ & $62.5 \%$ \\
\hline Grade II & $\mathrm{n}=11$ & 34.3 \\
\hline Grade III & $\mathrm{n}=1$ & $3.1 \%$ \\
\hline
\end{tabular}

\section{Discussion}

Spontaneous SAH has been classified into two types

1. Classical SAH.

2. Perimesencephalic SAH (PMSAH).

Classical SAH can be detected by the plain CT brain based on the thick blood shadows in the subarachnoid space which has the circle of willis. These patients usually are detected with a vascular structural abnormality. ${ }^{10-12}$

Perimesencephalic SAH on the other hand can be diagnosed on plain CT brain by the criteria which have been laid down and accepted by van Gijn. ${ }^{13}$ This was further detailed by Rinkel. ${ }^{14}$ Rinkel in his study of the distribution of subarachnoid haemorrhage with a normal angiogram in 52 patients described that, the center of the bleeding was located immediately anterior to the brainstem in all patients, extension to the ambient cisterns or to the basal parts of the sylvian fissures was common, but the lateral sylvian or anterior interhemispheric fissures were never completely filled with blood, rupture into the ventricular system did not occur. ${ }^{14,15} \mathrm{He}$ further comments that this pattern of SAH had a good prognosis. The study also mentions out of 221 plain ct scans given to two different radiologists 1 was wrongly labelled and was detected to have a basilar top aneurysm after the angiogram this proves that a follow up angiogram is a must to detect an abnormality. The incidence of angio negative SAH now referred to as PMSAH ranges from $2 \%-24 \%$. $^{2}$ It has an incidence of 0.5 per 100000 adult population. Flaherty et al in their study give a range of $5-10 \% .{ }^{16,17}$ In our study 32 patients were labelled PMSAH out of the 121 patients diagnosed with SAH that makes the incidence $26.4 \%$ which is on the higher side of the range. All patients presented to us in the first instance with headache, 3 had loss of conciousness, 8 had seizures and 1 had focal neurological deficit in the form of left hemiparesis which later recovered, the cause being vasospasm. 1 mortality due to sepsis was not taken as the direct cause due to SAH as there were variable reasons to the condition. The cause of PMSAH has been explained to be venous by Watanabe et al. In our study $2(6.25 \%)$ patients had features of vasospasm after the following DSA which was managed with calcium channel blockers nimodipine. They recovered within $24 \mathrm{hrs}$. All the patients are on regular follow up.

\section{Conclusion}

The entity of PMSAH had a good outcome in our study the incidence being $26.4 \%$ and the mortality was $1(3 \%)$ this being not directly due to the SAH. Based on the CT images and the criteria stated the patients can't be labelled PMSAH. A repeat vascular imaging at least 4 weeks after the primary ictus is necessary. A missed lesion will put the patient into a high risk of re-bleed which can be fatal. Effects of vasospasm must always be observed very carefully. Early intervention can reverse the effects. Maybe 3D recon of the imaging studies will help in detecting any hidden lesions. When in doubt a third opinion can be sought which will not cause harm to the patient. As of now further studies are required to find the cause of the bleed in PMSAH.

\section{References}

1. Julian S, R.L Macdonald, B Weir. Preoperative Management of SAH-Youmans Neurological Surgery 6th ed:363;3772-3790.

2. Kumar R, Das KK, Sahu RK, Sharma P, Mehrotra A, Srivastava AK, Sahu RN, Jaiswal AK, Behari S. Angio negative spontaneous subarachnoid hemorrhage: Is repeat angiogram required in all cases? Surg Neurol Int. 2014 Aug 7;5:125. 
3. Gupta SK, Gupta R, Khosla VK, Mohindra S, Chhabra R, Khandelwal N, et al. Nonaneurysmal nonperimesencephalic subarachnoid hemorrhage: Is it a benign entity? Surg Neurol. 2009;71:566-71.

4. Kaim A, Proske M, Kirsch E, von Weymarn A, Radü EW, Steinbrich W. Value of repeat-angiography in cases of unexplained subarachnoid hemorrhage (SAH) Acta Neurol Scand. 1996;93:366-73.

5. Yu DW, Jung YJ, Choi BY, Chang CH. Subarachnoid hemorrhage with negative baseline digital subtraction angiography: Is repeat digital subtraction angiography necessary? J Cerebrovasc Endovasc Neurosurg. 2012;14:210-5.

6. Drake CG. Report of World Federation of Neurological Surgeons Committee on a universal subarachnoid hemorrhage scale. J Neurosurg. 1988;68:985-6.

7. Fisher CM, Kistler JP, Davis JM. Relation of cerebral vasospasm to subarachnoid hemorrhage visualized by computerized tomographic scanning. Neurosurgery. 6 (1):1-9.

8. J M. Findlay, H R. Winn. Cerebral vasospasm- Youmans Neurological Surgery. 6th ed:364;3791-3800.

9. Soner Sahin, Emre Delen, Ender Korfali.

Perimesencephalic Subarachnoid haemorrhage: Etiologies, risk factors and necessity of second Angiogram. Asian J Neurosurg 2016 Jan-Mar;11(1):5053.

10. van Gijn J, Rinkel GJ. Subarachnoid haemorrhage: diagnosis, causes and management. Brain 2001 Feb;124(Pt 2):249-78.

11. Wardlaw JM, White PM. The detection and management of enraptured Intracranial aneurysm. Brain $2000 \mathrm{Feb}$; 123(Pt2):205-21.

12. Ahn SY, Lim DJ, Kim SH, Kim SD, Hong KS, Ha SK. Clinical analysis of patients with spontaneous subarachnoid hemorrhage of initial negative angiography. Korean J Cerebrovasc Surg. 2011;13:230-4.

13. Van Gijn J, Van Dongen KJ, Vermeulen M, Hijdra A. Perimesencephalic hemorrhage: A nonaneurysmal and benign form of subarachnoid hemorrhage. Neurology. 1985;35:493-7.

14. Rinkel GJ, Wijdicks EF, Vermeulen M, Ramos LM, Tanghe HL, Hasan D, et al. Nonaneurysmal perimesencephalic subarachnoid hemorrhage: $\mathrm{CT}$ and MR patterns that differ from aneurysmal rupture. AJNR Am J Neuroradiol. 1991;12:829-34.

15. Schwartz TH, Solomon RA. Perimesencephalic nonaneurysmal subarachnoid hemorrhage: Review of the literature. Neurosurgery. 1996;39:433-440.

16. Marder CP, Narla V, Fink JR et-al. Subarachnoid hemorrhage: beyond aneurysms. AJR Am J Roentgenol. 2014;202(1):25-37.

17. Flaherty ML, Haverbusch M, Kissela B et-al. Perimesencephalic subarachnoid hemorrhage: incidence, risk factors, and outcome. J Stroke Cerebrovasc Dis. 2005;14(6):267-71.

18. Watanabe A, Hirano K, Kamada M, Imamura K, Ishii N, Sekihara Y, et al. Perimesencephalic nonaneurysmal subarachnoid haemorrhage and variations in the veins. Neuroradiology. 2002;44:319-25. 